\title{
Etapas de la administración: hacia un enfoque sistémico
}

DOI: https://doi.org/10.21158/01208160.n87.2019.2412

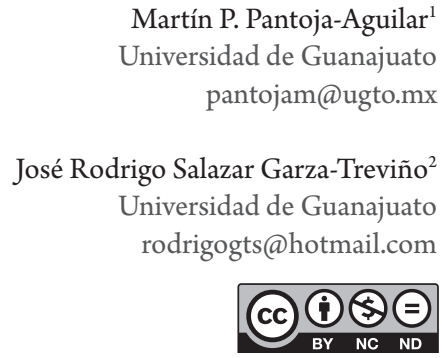

Cómo citar este artículo / To reference this article / Comment citer cet article / Para citar este artigo:

Pantoja-Aguilar, M. P.; Garza-Treviño, J. R. (2019). Etapas de la administración: hacia un enfoque sistémico. Revista Escuela de Administración de Negocios, (87), 139-154. DOI: https://doi.org/10.21158/01208160.n87.2019.2412

\section{Resumen}

El objeto de esta investigación se centra en homologar un análisis cronológico de las etapas de la administración hasta llegar a su actual enfoque sistémico. Se inicia con los precursores de la administración desde la civilización China, en la cual se sentaron las bases de un enfoque pragmático, y se abordan las diferentes etapas que condujeron a la humanidad hacia la revolución industrial cuando las máquinas sustituyeron a la mano de obra.

El artículo presenta un análisis crítico del surgimiento de la administración científica como una respuesta a la creciente demanda por estilos más humanos y sociales para ordenar la labor administrativa. Aplicando un análisis metodológico de cada etapa de las teorías de la administración bajo la visión de un enfoque sistémico, detectando las características que fueron aportando a la construcción de los nuevos modelos y su desenlace en los modelos actuales con enfoque sistémico, revisando desde los principios de la administración científica establecidos por Taylor, hasta la crítica que señalaba la falta de atención de los diferentes factores psicológicos y sociales relacionados con el ser humano.

Se concluye de esto que el nuevo enfoque sistémico es el resultado de un proceso de evolución de la teoría administrativa, así como del análisis de la adaptación y tropicalización de cada escuela administrativa con respecto a las necesidades de cada época, pero con una visión de futuro que conduce a una utopía con cierto grado de realismo.

Palabras clave: administración; historia de la administración; administración científica; teoría de la administración; escuela administrativa; modelos de administración; enfoque sistémico.

\footnotetext{
${ }^{1}$ Es Doctor en Liderazgo y Dirección de Empresas por la Universidad Anáhuac en México. Maestro en Administración con enfoque a Finanzas Corporativas por la Universidad de Shiga en Japón. Master of Business Administration (MBA) por la Southern Oregon University en los Estados Unidos de América. Contador Público por la Universidad de Guanajuato en México. ORCID: https:// orcid.org/0000-0002-8337-5427

${ }^{2}$ Licenciado en Comercio Internacional por la Universidad de Guanajuato en México. Es pasante de la Maestría en Administración reconocida por el CONACYT dentro del Programa Nacional de Posgrados de Calidad en la Universidad de Guanajuato en México. ORCID: https://orcid.org/0000-0003-2047-4695
} 


\title{
Stages of the administration: towards a systemic approach
}

\begin{abstract}
The purpose of this research is to standardize a chronological analysis of the stages of administration until reaching its current systemic approach. It begins with the precursors of the administration from the Chinese civilization in which the foundations of a pragmatic approach were laid and addresses the different stages that led humanity towards the industrial revolution when the machines replaced the labor force.

The article presents a critical analysis of the emergence of the scientific administration as a response to the growing demand for more human and social styles to organize the administrative work by applying a methodological analysis of each stage of the management theories under the vision of a systemic approach, detecting the characteristics that were contributing to the construction of the new models and their outcome in the current models with a systemic approach, reviewing from the principles of the scientific administration that were established by Taylor, until the criticism that indicated the lack of attention of the different psychological and social factors that are related to the human being.

From this, it can be concluded that the new systemic approach is the result of a process of evolution of the administrative theory, as well as of the analysis of the adaptation and tropicalization of each administrative school with respect to the needs of each era, but with a vision of the future that may lead to a utopia with certain degree of realism.
\end{abstract}

Keywords: administration; history of the administration; scientific administration; theory of the administration; administrative school; administration models; systemic approach.

\section{Etapas da administração: rumo a uma abordagem sistêmica}

\section{Resumo}

O objetivo desta pesquisa é homologar uma análise cronológica das etapas da administração até atingir sua atual abordagem sistêmica. Começa com os precursores da administração desde a civilização chinesa na qual foram lançadas as bases de uma abordagem pragmática, abordando as diferentes etapas que levaram a humanidade à revolução industrial, quando as máquinas substituíram o trabalho.

O artigo apresenta uma análise crítica do surgimento da administração científica como resposta à crescente demanda por mais estilos humanos e sociais para organizar o trabalho administrativo. Aplicando uma análise metodológica de cada estágio das teorias da administração sob a visão de uma abordagem sistêmica, detectando as características que contribuíram para a construção dos novos modelos e seus resultados nos modelos atuais com uma abordagem sistêmica, revisando os princípios de a administração científica estabelecida por Taylor, até as críticas que indicaram a falta de atenção dos diferentes fatores psicológicos e sociais relacionados ao ser humano.

Conclui-se que a nova abordagem sistêmica é o resultado de um processo de evolução da teoria administrativa, bem como da análise da adaptação e tropicalização de cada escola administrativa em relação às necessidades de cada época, mas com uma visão de futuro o que leva a uma utopia com algum grau de realismo.

Palavras-chave: administração; história da administração; administração científica; teoria da administração; escola administrativa; modelos de administração; abordagem sistêmica. 


\section{Vers une approche systémique des processus de gestion}

\section{Résumé}

Cette investigation a pour but de normaliser une analyse chronologique des processus de gestion pour atteindre l'approche systémique actuelle. Les recherches remontent aux précurseurs de l'administration chinoise posant les fondements d'une approche pragmatique, et les processus ayant conduit l'humanité vers la révolution industrielle où les machines ont progressivement remplacé le travail des hommes.

Cet article présente une analyse critique de l'émergence de la gestion scientifique en réponse à la demande croissante d'approches plus humaines de la gestion des tâches administratives. Notre étude offre une analyse méthodologique des théories de gestion systémique, en montrant les caractéristiques contribuant à la construction de nouveaux modèles et de leurs résultats, et en s'appuyant sur les principes de gestion scientifique établis par Taylor qui mettent en évidence le désintérêt apporté aux facteurs psychologiques et sociaux de l'être humain.

L'investigation conclut que l'approche systémique est le résultat d'un processus d'évolution des théories de gestion, de l'analyse de l'adaptation et de la tropicalisation des écoles de gestion en rapport aux besoins de chaque époque pouvant conduire à une certaine utopie teintée de réalisme.

Mots-clés: gestion; histoire de la gestion; gestion scientifique; théorie de gestion; école de gestion; modèles de gestion; approche systémique. 


\section{Introducción}

$\mathrm{L}$ a administración forma parte integral de las actividades que realiza el ser humano a fin de convivir y lograr sus objetivos personales y los que elige establecer en sociedad. Desde sus inicios ha sido pieza fundamental en el comportamiento $y$ las actividades del ser humano y, por tanto, ha tenido diversas etapas de desarrollo que han recibido fuertes críticas, así como ha generado grandes beneficios. Es por esto que el análisis cronológico de las etapas de la administración, desde sus inicios hasta que se convierte en ciencia, resulta atractivo para comprender los enfoques modernos y mejorar de manera continua en su aplicación y desarrollo. La metodología utilizada en el presente artículo consistió en una revisión crítica de cada una de las etapas de la teoría de la administración con el propósito de detectar los elementos especiales que originaron los cambios en cada enfoque, los cuales construyeron de manera progresiva la base de las actuales teorías de la administración con un enfoque sistémico. La revisión detallada de los autores clásicos generó la base que permite comprender cómo y por qué se llegó a los nuevos modelos sistémicos y, por tanto, nos brinda un marco de referencia para futuras investigaciones sobre las posibles causas de éxito o fracaso en la implementación de dichos modelos. Los inicios de la administración contenían principios que el ser humano ha adoptado a lo largo de siglos, las teorías modernas de la administración los postulan aún y los administradores modernos siguen utilizándolos. Así mismo, se encuentran aspectos característicos que han sido innovados a fin de lograr la comprensión del funcionamiento administrativo de las organizaciones, los cuales ayudan a establecer nuevos paradigmas en busca de la mejora continua y la innovación de los procesos - y, por ende, el bienestar del ser humano-.

En este proceso histórico del ser humano y la administración se pueden agrupar las distintas teorías de la administración con base en dos grandes etapas por las que ha transcurrido la existencia del hombre moderno: la mecánica y la de sistemas. La primera es la etapa que se enfoca en los procesos mecánicos establecidos de manera ordenada e inorgánica por el ser humano. La segunda es la que se enfoca en los procesos naturales y orgánicos sobre los que reflexiona e identifica el ser humano al comprender mejor su contexto y su ambiente.

De las últimas dos eras en la civilización humana forman parte la era de la mecanización y la reciente llamada «era de los sistemas». Según Ackoff (2012), nuestra civilización está saliendo de la era denominada «de la máquina»y, sin estar en capacidad de determinar un punto de partida exacto, hemos iniciado, desde finales del siglo XIX, la era «de los sistemas». No obstante, algunos investigadores toman como fecha formal 1969, a partir de la publicación del libro Teoría general de sistemas de von Bertalanffy. En ambas etapas la organización ha sido el centro de su aplicación. Herrscher define esta última como «estructuras sociales creadas por individuos para apoyar la búsqueda en colaboración de objetivos específicos» (2009, p. 51). Estos objetivos se pueden identificar por los elementos orgánicos e inorgánicos que el ser humano ha integrado de manera progresiva a las prácticas, o las teorías que utiliza para organizarse y llegar a mejorar en la búsqueda del bienestar personal y el común.

La denominada «era de la máquina», que abarca desde las primeras civilizaciones hasta finales de la década de los sesenta del siglo XX, se basa en los principios del análisis - método básico de investigación-, el reduccionismo y el determinismo. Lo anterior, al concebir el análisis como la desintegración de un todo para entender la función general de un objeto a partir del desempeño y la conducta de las partes, esto es, partir de las partes para comprender el todo. Por otro lado, el reduccionismo parte de la idea según la cual un todo puede analizarse al descompo- 
nerlo en sus partes constitutivas. De esta manera, los sistemas de un nivel son integrantes de sistemas más fundamentales que, a su vez, son parte de elementos fundamentales irreductibles. Por último, el determinismo basa su paradigma en el hecho de que a todo efecto precede una causa única, sin tomar en cuenta el medio en el cual existe la causa-efecto. Las tres etapas que integran el proceso de análisis, y que Ackoff (2012) menciona, son: 1) separar las partes; 2) comprender el comportamiento de las partes; y 3) reunir este entendimiento en una comprensión del todo. En esta etapa del ser humano, las teorías de la administración se han enfocado en aplicar elementos inorgánicos que buscan establecer un orden por mandato y por decisión unilateral, lo cual bajo un contexto inanimado puede funcionar y ser productivo, pero genera poco a poco un costo social y humano que a la larga produce un impacto explosivo. Lo anterior, al crear un ambiente hostil, de múltiples implicaciones de convivencia, que deteriora la calidad de vida del ser humano y lo lleva a perder el valor social creado, de modo que contraviene el objetivo principal de las organizaciones.

Por su parte, la reciente y aún en formación era de los sistemas surge como consecuencia del cambio de visión en el mundo, y como respuesta a diversos dilemas generados por el mecanicismo operante en siglos pasados. El pensamiento sistémico plantea la complementariedad con los postulados del mecanicismo, pero con una visión más general y externa, y, por tanto, sistémica. De acuerdo con Arias, Del Toro y Guerrero,

la teoría administrativa ha abandonado paulatinamente la visión del método analítico a ultranza y con este, el reduccionismo y el determinismo que le son afines, al pensar al mundo, ya como un conjunto de partículas elementales, esenciales, o bien, como una cadena de causas y efectos que se suceden y se recrean a sí mismos en una cadena interminable. (2013, p. 1616)

Esto es, la generación de conocimiento administrativo enfocado en un orden orgánico al que la perfecta unión de esfuerzos multiplicadores supera, y el cual, al beneficiar el bien personal, logra multiplicar los efectos del bien común.

Según Hernández, «la teoría moderna de sistemas se desarrolló gracias a Ludwing von Bertalanffy (1969), quien señaló que no existe elemento físico o químico independiente; todos los sistemas están integrados en unidades relativamente interdependientes». (2008, p. 63) El pensamiento sistémico centra su paradigma en una visión integradora, de modo que les da mayor importancia a las interacciones de las partes integrantes de un todo —visión sistémica- que a las interpretaciones de las partes consideradas de manera separada o individual. Aunado a esto, se incluye el concepto de los sistemas y los suprasistemas, al entender que el análisis de un sistema siempre depende de su relación con un sistema mayor, o suprasistema, y que no se ve limitado de forma dialéctica por el pensamiento reduccionista existente en el mecanicismo.

Por tanto, un sistema se entiende como el conjunto que integran dos o más elementos en el que: a) el comportamiento de cada una de las partes tiene un efecto en el comportamiento del sistema; b) existe interdependencia entre el comportamiento de las partes y el efecto que producen en el sistema, $y$ c) no puede existir independencia de subgrupos formados al interior del sistema con respecto a su efecto sobre el mismo (Ackoff, 2012). Es por esto que en una organización la suma de las partes puede generar un efecto multiplicador en el logro de los objetivos buscados y que beneficia de manera más contundente y equitativa a todos sus integrantes, de manera que favorece un ambiente superior al orgánico que perfecciona la naturaleza de la organización.

El proceso que sigue en sus tres etapas el análisis en el enfoque sistémico es: 1) identificar un todo contenedor - sistema- del cual el objeto por explicar es una parte; 2) explicar el comportamiento o las propiedades del todo o contenedor; y 3 ) explicar el comportamiento o las propiedades del objeto por explicar en términos de su(s) papel(es) 
o función(es) dentro de su todo o contenedor (Ackoff, 2012). Bajo este enfoque, las organizaciones se vuelven un todo integrador de subsistemas que trabajan en conjunto, pero más aun al multiplicar los efectos de su impacto en cada una de las unidades que integran cada subsistema. Así mismo, al replicar los impactos multiplicadores en los otros subsistemas. Este enfoque de trabajo genera un desarrollo innovador que garantiza la permanencia y la mejora de las organizaciones que logran establecer este enfoque en su administración.

\section{Las primeras etapas y su desenlace en la Revolución Industrial}

$\mathrm{E}$ n sus inicios, la era mecanicista de la administración tuvo varias etapas que adoptaron una ideología de los negocios enfocada en el comercio: «Algunos de nuestros iniciales conceptos de administración se encuentran en $\mathrm{El}$ arte de la guerra, escrito hace más o menos 2500 años por Sun Tzu, un general chino» (Gómez-Mejía, Balkin y Crady, 2008). Por su parte, Bateman y Snell plantean que «alrededor del año 1100 a. C., los chinos practicaban las cuatro funciones de la administración [...]: la planeación, la organización, el liderazgo y el control» (2009, p. 41).

Luego pasa la administración por diversas etapas, relacionadas con las civilizaciones antiguas. Inicia con los judíos y las civilizaciones de Asia Menor que contenían reglas base para la administración moderna; los griegos con su influencia a través de la mitología y la disciplina militar; los romanos con la promulgación del derecho público y la creación del concepto de «persona moral»; la Iglesia católica con el modelo de estructura que siguieron las empresas modernas al usar la división de la línea funcional; la época medieval con la creación de los conceptos de «vida civil»y «vida espiritual»; y el Renacimiento que sentó las bases del capitalismo moderno mediante la justificación de la acumulación de capital para la realización de negocios.

Posteriormente a estas etapas, una escalada de nuevos inventos, principalmente, máquinas que suplían la mano de obra y mejoraban los ritmos de producción, tuvo origen, primero, en Gran Bretaña, y después en muchos países de Europa.
Los pequeños talleres dirigidos por trabajadores calificados, que hacían a mano los productos un sistema llamado de producción artesanal-, comenzaron a ser sustituidos por fábricas grandes en que máquinas complejas controladas por cientos y aún miles de trabajadores no calificados -o poco calificados - hacían los productos. (Jones y George, 2010, p. 42)

Bajo este enfoque administrativo la consideración de factores personales y sociales del ser humano se dejaron de lado. Se identificaba al trabajador como uno más de los elementos inorgánicos que solo requería de un salario para otorgar su esfuerzo a cambio. No se consideraban los elementos sociales y personales que afectan el desempeño de las personas, sino que se consideraba que el costo mínimo compensaba el desgaste de la planta laboral.

En contraste con esta tendencia, Robert Owen (1835) se considera el principal pensador del socialismo en la administración, ya que llevó a la práctica varias de sus ideas y mediante hechos concretos demostró que era factible obtener utilidades y estar en capacidad de otorgar a los trabajadores prestaciones importantes, como, por ejemplo, el otorgamiento de vivienda, escuela para los hijos y comedores, por citar algunos ejemplos (Hernández, 2008). Esta tendencia fue la base para generar una nueva discusión que estableciera un enfoque más humano que tomara en cuenta la cobertura de las necesidades de los trabajadores y su relación con el nivel de producción y eficiencia organizacional. 


\section{Surgimiento de la administración científica}

$\mathrm{D}$

e acuerdo con Hernández,

Se le ha designado a esta época de la evolución del pensamiento administrativo como la etapa científica debido a que a finales del siglo XIX y principios del XX, diversos autores se mostraron interesados en investigar "científicamente" la problemática que presentan las empresas industriales como la producción masiva y estandarizada. (2008, p. 33)

Como antecedente de la administración científica se pueden citar las ideas de Adam Smith, las cuales contribuyeron al establecimiento de la denominada «administración sistemática»: «La administración sistemática enfatizaba las operaciones internas por que los administradores se preocupaban principalmente de cumplir con el crecimiento explosivo de la demanda generada por la Revolución Industrial» (Bateman y Snell, 2009, p. 42).

El enfoque científico toma fuerza con el estadounidense Frederick Winslow Taylor (1911), quien al ver que la administración sistemática no mejoraba la eficiencia de la producción concluye que las decisiones administrativas no son sistemáticas y deben, por tanto, diseñarse científicamente (Certo, 2001). En este sentido realiza su principal aportación a través del estudio de «tiempos y movimientos», basado en los principios de especialización y división del trabajo promulgados por Adam Smith (Chiavenato, 2005). Según Ramírez, «Taylor afirmó que cada operación administrativa debe racionalizarse de tal manera que esta pueda ser ejecutada científicamente $\gg(2009$, p. 129).

Después de Taylor, iniciado el siglo XX, surgieron otros pensadores que basaron sus paradigmas en la escuela del primero. El matrimonio formado por Frank y Lillian Gilbreth en la primera década del siglo XX aplicó y mejoró la técnica de tiempo y movimientos al enfocar aún en mayor grado la división del trabajo en su mínima expresión posible y tomar acciones en lo específico (Hampton, 2003).
En la misma década, el socio de Taylor, Henry Gantt, creó la famosa «gráfica de Gantt» basada en una serie de barras que miden el trabajo planeado contra el terminado en series de tiempos (Daft, 2004). Por su parte, Henry Ford introdujo en 1913 el concepto de la banda transportadora y, al exagerar la aplicación de los principios de la administración de Taylor, llevó a cabo un sistema de producción y de administración similar al de una máquina. En esa misma década, «MaxWeber desarrolló los principios de la burocracia durante la revolución industrial en Alemania, para ayudar a las organizaciones a elevar su eficiencia y eficacia» (Jones y George, 2010, p. 50 ), de manera que fue este un modelo muy sensible a los efectos del tipo de liderazgo y al compromiso real de los directivos, en especial para la época en la que se implementó dicho pensamiento.

Al consolidar los principios emitidos por sus antecesores, Henry Fayol, a inicios de la segunda década del siglo $\mathrm{XX}$, declaró que «la función administrativa tiene su única manifestación a través de los miembros de la organización -cuerpo social—» (como se cita en Merril, 1999, p. 185), y, de manera precisa,

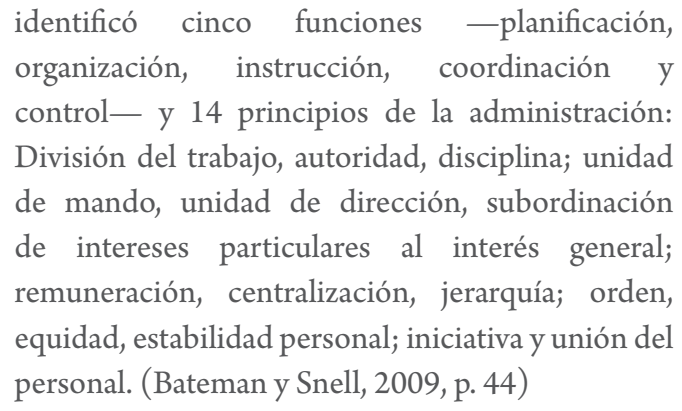

A Fayol se le considera el padre del proceso administrativo, además de la división de las áreas funcionales delas empresas. Varios de estos principios establecidos por Fayol son aún vigentes y se aplican en los enfoques más modernos de las organizaciones y su administración. Principios como, por ejemplo, la unión del personal, la iniciativa y la estabilidad 
personal son todavía fundamentales en las teorías modernas, e incluso en el enfoque sistémico. Sin embargo, otros principios como, por ejemplo, los de centralización y la jerarquía han sido modificados y no se aplican, hoy en día, de la manera tradicional en que fueron propuestos por su autor, sino que han evolucionado a una forma más orgánica y adecuada al ámbito organizacional moderno que busca ser parte de un suprasistema sustentable y eficiente.

\subsection{La escuela cuantitativa}

Dentro de la administración científica, la escuela cuantitativa tuvo su origen en los diferentes pensadores que usaron modelos matemáticos en las operaciones de las empresas con base en las ideas de Taylor (Hernández, 2008). Walter A. Shewhart (1924) diseñó el método de calidad al establecer los cuatro pasos del control: 1) fijar estándares de calidad; 2) operar con los estándares; 3) actuar cuando se excede el límite superior o inferior de los estándares; y 4) hacer correcciones. Entre los pensadores principales de esta tendencia se encuentran Ansoff, Declerk y Hayes (2003), Arnoff (1971), Churchman (1971) y Ackoff (2012). De aquí el origen de lo que posteriormente será el enfoque sistémico de Ackoff basado en la técnica de la investigación de operaciones. Esta escuela constituye una sólida base de principios que en la administración moderna han tenido resultados beneficiosos, los cuales se integran a la organización de manera cotidiana. Sin embargo, las organizaciones modernas y sus líderes todavía se debaten por encontrar la manera de sincronizar los objetivos y las actitudes personales con las bondades del desempeño organizacional bajo un enfoque científico.

\subsection{Los enfoques contemporáneos y los humanistas}

Los enfoques contemporáneos de la administración científica surgieron como respuesta a la búsqueda de mejores resultados afectivos y de satisfacción personal en las organizaciones. Con la base establecida por la escuela cuantitativa, el reto era encontrar un enfoque que permitiera beneficiar a la organización a través de la satisfacción de las necesidades humanas y sociales de sus colaboradores. Dentro de los enfoques contemporáneos, a principios de la década de los ochenta del siglo XX, Tom Peters y Robert Waterman identificaron con su propia metodología tres conjuntos de principios relacionados, con frecuencia aplicados por los directivos de las empresas y que serían la base del enfoque sistémico planteado por pensadores como Ackoff: 1) autonomía de gestión y espíritu emprendedor con la descentralización de la autoridad; 2) plan maestro y enfoque en mejorar los bienes y servicios centrados en el cliente; y 3) división del trabajo, de la autoridad y de la responsabilidad al subordinar intereses personales al interés común (Jones y George, 2010). Estos principios fueron fundamentales en las teorías desarrolladas luego y que buscaron darle un tinte personalizado a la satisfacción de las necesidades humanas y sociales de los integrantes de una organización al enfocarse en una dimensión en particular.

Un ejemplo claro fue la estadounidense Mary Parker Follet, quien criticó de manera abierta el enfoque de Taylor, ya que «ignoraba muchos de los factores sociales y psicológicos relacionados con el trabajo, resaltando únicamente el dinero como incentivo del trabajador» (Bateman y Snell, 2009, p. 44). Varela por su parte, reconoce que,

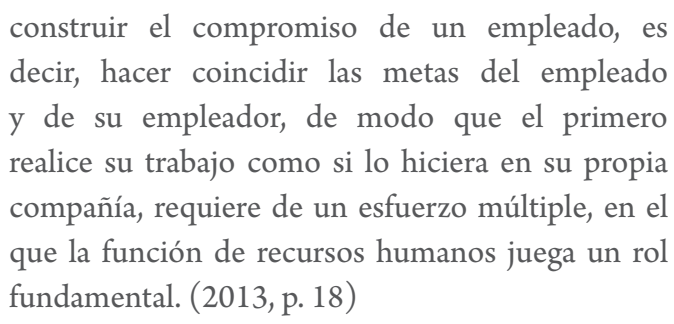

De acuerdo con Bateman y Snell «dos de [las] contribuciones más importantes [de Follet fueron]: la noción de que los administradores desean flexibilidad y las diferencias entre motivar a grupos y a individuos, prepararon el terreno para la perspectiva moderna de la [administración por] contingencia» (2009, p. 44). Estas dos contribuciones se identifican como la descentralización y el trabajo en equipo que posteriormente retoma la escuela con enfoque en sistemas. 
Otro gran aporte al pensamiento sistémico lo realizó el psicólogo Elton Mayo, quien entre 1924 y 1927, a través de los estudios de Hawthorne - barrio en el que se ubica entonces la empresa Western Electric- detectó que las condiciones físicas del lugar de trabajo no tienen tanta influencia en la productividad como lo tienen los factores sociales, así como los efectos del comportamiento grupal al interior de la organización (Koontz y Weihrich, 1998). Por su parte, Abraham Maslow creía que los gerentes deben entender las necesidades fisiológicas, de seguridad, sociales, de estima y de actualización que los miembros de la organización necesitan (Certo, 2001). La aportación científica del investigador Frederick Herzberg abona a favor de los principios de la organización sistémica actual, ya que el investigador sostuvo en sus estudios según comenta Hernández (2008) — que más allá del salario y las prestaciones, el principal factor motivacional del trabajador es la libertad para tomar decisiones y de ser creativo.

Por su parte, en la década de los sesenta del siglo $\mathrm{XX}$, Douglas McGregor propuso la teoría X y la teoría Y. Estableció la existencia de un patrón de conducta en los directivos que fungen de acuerdo con la teoría $\mathrm{X}$ como líderes mecanicistas que no confían en las personas y consideran que solo a través de la supervisión y la imposición de normas estrictas, adicionadas a la práctica de la técnica de estímulo-respuesta, pueden hacer que los objetivos de la organización se alcancen. En cuanto a la teoría Y, estableció la existencia de un directivo con enfoque de líder que confía en los trabajadores, los considera suficientemente maduros para tomar el reto de cumplir con las metas de la organización y delegar en ellos la toma de decisiones; esto contiene pensamiento sistémico y una conciencia de grupo (Chiavenato, 2005).

Para la década de los noventas del siglo XX, Robert Blake y Jane S. Mouton crearon un modelo graficado denominado «malla»o « grid gerencial», en el cual mostraban las distintas combinaciones que puede tener un líder al balancear, mediante su enfoque administrativo, las necesidades humanas de las personas y la atención a la producción. Como se puede observar, las variaciones de enfoques eran aún determinadas por aspectos parciales de la administración dentro del contexto de la escuela científica y, cada vez más, se acercaban a la comprensión de la teoría del todo organizacional, de modo que se complementaban con cada aspecto abordado. Esto es, la llegada al enfoque integrador que consideraba las diferentes dimensiones de los aspectos organizacionales, sus impactos y sus retos, todos considerados dentro de un sistema.

\subsection{El enfoque en los sistemas y en la contingencia}

Según Kast y Rosenzweig «muchos científicos de la conducta destacan el valor de organizaciones estructurales de manera más democrática, menos autoritaria y menos jerarquizada que las propuestas por el enfoque tradicional» (1987, p. 89). De acuerdo con Certo (2001), la teoría moderna de sistemas se desarrolló gracias a Karl Ludwig von Bertalanffy. Se aplica a la administración mediante la premisa según la cual la organización es un sistema y se debe comprender la interdependencia de sus partes. Con relación a esto, Fretes menciona que «así como en la naturaleza, los organismos vivientes, las empresas humanas entre ellas los negocios, son sistemas $\gg$ (2009, p. 6); añade Garza: «Se ha definido un sistema como un todo unitario organizado, compuesto por dos o más partes, componentes o subsistemas interdependientes $y$ delineado por límites identificables de su ambiente o suprasistema» (2003, p. 29).

Por su parte, Gily Giner mencionan que «la organización tiene como rasgo fundamental constituir una entidad social, que se estudia a diferentes niveles, según la escala de sistemas humanos que considere» (2012, p. 57). Tal como lo afirmara en su teoría sobre sistemas Johansen, «un objeto posee sinergia cuando el examen de una o alguna de sus partes incluso a cada una de sus partes - en forma aislada, no puede explicar o predecir la conducta del todo» (2004, p. 36). 
$\mathrm{Al}$ aplicar el concepto de sinergia a la administración, Ackoff afirma — con relación a la iteración del sistema y sus partes - que «cualquier cambio o subconjunto de cambios puede mejorar de manera significativa el desempeño organizacional. Sin embargo, cuando todos se llevan a cabo juntos, hay un poderoso efecto multiplicador, que es mucho mayor que la suma de sus partes» (2012, p. 43). Como lo señala Csikszentmihalyi,

quizás la consecuencia más importante del modelo de sistemas es que el grado de creatividad presente en un lugar y un tiempo determinados no depende sólo de la cantidad de creatividad individual. También depende en igual medida de lo bien dispuestos que estén los respectivos campos y ámbitos para el reconocimiento y difusión de ideas novedosas. (1998, p. 118)

Kast y Rosenzweig consideraron que,

la organización puede ser considerada en términos de un modelo de sistema abierto general, $[. .$.$] en$ constante interacción con su medio ambiente y logra un "estado estable" o equilibrio dinámico, al tiempo que retiene la capacidad para trabajar o la transformación de energía. (1987)

En la administración contemporánea con enfoque en los sistemas resalta la figura de Peter F. Drucker (como se cita en Hernández, 2008), quien explica que «la mayor ventaja de la dirección mediante objetivos es, quizá, que hace posible que un subordinado controle sus propias realizaciones: el autocontrol significa mayor automotivación» (p. 78). Russell L. Ackoff (2012), como pensador del campo administrativo, mostró un nuevo paradigma al rescatar los avances científicos que en el análisis de los sistemas se habían realizado, con lo cual se convirtió en uno de los pioneros en el análisis de las organizaciones mediante sus operaciones y los sistemas.

Con las distintas corrientes actuales del pensamiento administrativo surge con mayor fuerza la idea según la cual

conforme avanzan las ideas sobre la teoría de sistemas, la perspectiva de las contingencias refuta los principios universales de la administración, declarando que un sinfín de factores, tanto internos como externos a la compañía, pueden afectar el desempeño de la organización. (Bateman y Snell, 2009, p. 47)

Chiavenato menciona que, «según esta teoría, no existe nada universal ni definitivo en administración pues todo es relativo y depende del contexto ambiental» (2005, p. 69).

La teoría de las contingencias la desarrollaron, en 1960, los británicos Tom Burns y G. M. Staker, así como los estadounidenses Paul Lawrence y Jay Lorsch. Comenta Certo que «en general, el enfoque de las contingencias, también llamado situacional, intenta bosquejar las condiciones o situaciones en que varios métodos administrativos tengan la mayor probabilidad de éxito» (2001, p. 36). Por su parte, señala Velásquez que «la contingencia reconoce similitudes y diferencias entre organizaciones, pero mantiene énfasis en que el papel primario gerencial es el de buscar congruencia entre sus subsistemas» (2000, p. 36). Con esta teoría se reafirma lo que, posteriormente, se denomina «planeación interactiva $\gg, y$ se establece como parte de un sistema efectivo. Concluyen López, Arias y Rave:

\footnotetext{
Los aportes que han hecho a las organizaciones para su actuar gerencial y su desempeño efectivo en los diferentes enfoques muestran cómo a través de un trabajo grupal y armónico, eficaz y eficiente, e interactuando con el ambiente se logra productividad y competitividad en un mundo cambiante y exigente. (2006, p. 152)
}

\subsection{La aplicación de la teoría de los sistemas a la administración}

«La teoría de los sistemas aplicada a la administración hace énfasis en la interdependencia entre las distintas partes de una organización, la organización misma y su medio» (Eyssautier, 2002). Por eso, «concebir a la organización como una red de grupos interrelacionados ayuda a ver que el desempeño global depende no sólo de la eficacia con que cada grupo efectúe su trabajo, sino también de la eficacia de su interrelación» (Hampton, 2003, p. 266). 
Ackoff (2012) parte de una tipificación inicial de los sistemas, y a su vez de los modelos organizacionales en: deterministas, animados, sociales y ecológicos. Para esto utiliza la premisa según la cual cada sistema contiene a otro sistema como parte del él mismo, y al final, todos los sistemas los contiene un suprasistema; así mismo, aplica el análisis de las partes y el todo con base en su intencionalidad.

De acuerdo con Hellriegel, Jackson y Slocum, «en un sistema mecanicista [determinista] la gerencia descompone las actividades en tareas separadas y muy especializadas, depende mucho de normas y reglas y centraliza la toma de decisiones en la cima» (2010, p. 376). Ackoff (2012) lo identifica como un sistema no intencionado, así como sus partes. En este ámbito de las organizaciones, Mintzberg ubica y describe las burocracias como aquellas que «su trabajo operativo es rutinario, en su mayor parte bastante simple y repetitivo, como resultado, sus procesos de trabajo son altamente estandarizados» (2001, p. 197). Posteriormente, Ackoff (2012) describe el tipo «animado» de organización en el cual sus partes sí son intencionadas pero el todo no lo es y contiene entre sus partes otros sistemas deterministas - por ejemplo, los animales-. Hellriegel et al. (2010) describen este modelo como orgánico, en el cual los administradores y los subordinados trabajan juntos en equipos y se comunican entre sí de manera abierta.

Los modelos sociales tienen una intencionalidad como un todo, pero sus partes también tienen intencionalidades $\mathrm{y}$ contienen entre sus partes sistemas animados (Ackoff, 2012). Además, por lo general, forman parte de sistemas sociales más grandes (p. ej., la universidad). Al final se citan los modelos del tipo «ecológico», los cuales no son intencionados como un todo, pero sus partes sí lo son. Este tipo de sistema siempre contiene a los otros tres tipos mencionados, de modo que interactúan entre sí y es un suprasistema (Ackoff, 2012) (p. ej., la tierra). Para Argyris, en este sentido, «las organizaciones aprenden a través de los individuos que actúan como agentes de ellas. Las actividades de aprendizaje del individuo, a su vez, se facilitan o se inhiben mediante un sistema ecológico de factores que podríamos llamar sistema de aprendizaje organizacional» (2001, p. 234).

De acuerdo con Kendall y Kendall, «las organizaciones se consideran sistemas diseñados para cumplir metas y objetivos predeterminados con la intervención de la gente y otros recursos de que disponen» (2005, p .27). Cuando se aplican modelos deterministas -mecanicistas - a las organizaciones, se pueden generar buenos resultados solo cuando el sistema está poco desarrollado y las partes del sistema se consideran por separado. Un ejemplo de esto sería la burocracia que necesita de la centralización de las decisiones, pero, al tratarse de organizaciones complejas, la descentralización se vuelve necesaria.

Cuando se aplican modelos animados — también llamados «organicistas»- a las organizaciones, se establece una semejanza con el cuerpo humano, en la que el cerebro es la administración y el cuerpo es la parte operativa de la organización. La estructura funciona como un robot que realiza las instrucciones detalladas que la administración central genera y que se siguen sin desviaciones. La aplicación del modelo social-sistémico a las organizaciones reconoce a sus partes como unidades poseedoras de intenciones y se basa en una supervisión de las interacciones entre sus elementos y con el exterior, $y$ no de las acciones de estos, de modo que se maneja la solución de conflictos connatural a las organizaciones que permiten la elección entre sus partes. Todo esto es posible cuando se trata de organizaciones con un alto grado de desarrollo (Ackoff, 2012).

Es importante detectar en este modelo la existencia del concepto de sistema emergente, el cual Johnson describe como los que «resuelven problemas recurriendo a masas de elementos relativamente no inteligentes en lugar de hacerlo recurriendo a un solo 'brazo ejecutor' inteligente. Son sistemas ascendentes no descendentes. Extraen su inteligencia de la base» (2003, p. 19). Las características propuestas para un modelo organizacional social-sistémico y que no le son aplicables a los otros modelos son: 
organización democrática, economía interna de mercado, estructura organizacional multidimensional, planeación interactiva y sistema de apoyo a las decisiones (Ackoff, 2012).

Entre estas características, a manera de ejemplo, podemos citar los hallazgos de Contreras y Benítez (2011), quienes demuestran que el proceso de toma de decisiones cambia también en relación con el tamaño de la organización. Cuando la organización es pequeña o inicia, las decisiones son intuitivas e informales, y cuando la organización crece y se desarrolla tiende a tomar decisiones de manera más colectiva y formal. Para esto, la cultura juega un papel importante al crear un sentido de identidad compartida y genera en los miembros de la organización un compromiso más allá de los intereses personales (Gómez-Mejía et al., 2008). En los últimos años este tipo de administración ha sido identificada como «de enfoque socioeconómico de gestión», el cual, según Savall, Zardet y Bonnet, «fue creado para estimular el ritmo de transformación adecuado de las empresas y organizaciones para adaptarse a un nuevo entorno altamente competitivo y mejorar su desempeño ante los actuales desafíos económicos» (2008, p. 16). Al reflejar esta condición en una organización, Bartol y Martin (1998) establecían la existencia de cuatro diseños principales en la estructura horizontal de las organizaciones: funcional, divisional, híbrida y matricial. De acuerdo con Druker, «las organizaciones se construyen sobre la confianza, y la confianza se basa en la comunicación y el entendimiento mutuo» (2006, p. 361). Eso supone al ser humano como centro del modelo sistémico y, de acuerdo con Bolman y Deal, desde hace ya décadas que las «organizaciones existen para servir a las necesidades humanas - y los humanos no existen para servir a las necesidades de la organización—》(1984, p. 65).

\subsection{La administración sistémica aplicada}

Según Debernardo y Hurtado, «el pensamiento sistémico nació hace más de cincuenta años. Sin embargo, aún muy pocas organizaciones en el mundo son gestionadas dentro de este paradigma» (2006, p. 13). Entendamos por gestión «un movimiento histórico de renovación y racionalización de los artefactos institucionales para la acción colectiva» (Meléndez et al., 2010, p. 216).

El diseño de un sistema de administración debe reflejar una estructura organizacional, un estilo administrativo y un liderazgo, orientados hacia un tipo de sistema y modelo que coincidan en enfoque y permita no solo el crecimiento, sino, más aún, su desarrollo. En este sentido, «es necesario [concebir la organización] como un desarrollo holístico desde el punto de vista de los conceptos del enfoque sistémico para el diseño organizacional, las métricas y las mejoras de procesos» (Ahoy, 2010, p. 188). El diseño de una administración sistémica debe considerar las demandas actuales de un mundo cambiante, y su característica principal debe ser la transformación constante derivada de los cambios internos o externos —adaptación—, así como el autoaprendizaje-aprendiendo a aprender- que le permita dar respuesta interactiva a los retos que se le presenten, de manera que se reconozca como un sistema intencionado con posibilidades de desarrollo.

De acuerdo con Ríos y Mustafá, «se puede afirmar que en los sistemas administrativos se presentan relaciones de funcionalidad similares a las encontradas en la naturaleza misma» (2004, p. 178). Ansoff et al. (2003) declaran que las organizaciones se relacionan con su medio ambiente de dos maneras: a través del comportamiento competitivo operación normal y diaria- $-\mathrm{y}$ a través del comportamiento estratégico — reemplazo de objetos e ideas obsoletos por innovadores-. Etkin identifica las organizaciones como viables al relacionarlas con «la posibilidad de autocontrol, esto es, de generar desde dentro las medidas correctivas de los comportamientos para enfrentar una realidad cambiante» (2005, p. 59).

El entorno actual demanda la existencia de la organización que aprende, la que «enfatiza la solución sistemática de problemas, la experimentación con nuevas ideas, el aprendizaje de la experiencia y de la 
historia anterior, el aprendizaje de experiencias ajenas, y la rápida transferencia de conocimiento a través de la organización» (Certo, 2001, p. 40). Esta visión de la organización concuerda, en esencia, con el enfoque sistémico de la administración moderna. Majluf se refiere a la organización moderna, la de hoy, y afirma que «en una economía del conocimiento, es una organización que aprende. Su foco de atención no está en hacer las cosas más eficientes que su competencia, sino en prender más rápido» (2011, p. 110).

Una herramienta útil en las labores de los administradores es el análisis estructural que «demanda trabajar con modelos sistémicos que, al valorar de manera cualitativa a los elementos del sistema, permiten al administrador identificar los tipos de relaciones que se dan entre estos y así acceder a conformar la toma de decisiones sustantivas para la organización, permitiendo optimizar los recursos de la misma» (Valdés, 2005, p. 149).

\subsubsection{El diseño general de un sistema de administración.}

Si bien Ackoff (2012) propone un diseño de administración basado en el enfoque sistémico y describe las principales características que lo integran, aclara que el diseño propuesto es esquemático y general, por lo cual toda organización deberá crear el diseño ideal a partir de estos elementos, pero incluir las variantes que la propia organización estime convenientes. Las características principales de una administración sistémica descritas por Ackoff (2012) son: a) autonomía aprender a aprender y capacidad de adaptación-; b) reconoce la interacción de la organización misma, sus unidades y el entorno; c) consta de cuatro subsistemas: 1) diagnóstico y prescripción, 2) toma de decisiones, 3) mejoramiento y mantenimiento del desempeño; y 4) el subsistema de información; d) se identifican desviaciones, amenazas y oportunidades, y síntomas y presíntomas; e) diseño de diversas unidades que requieren instancias de coordinación e integración; f) coordinación e integración a través de consejos para la asignación de decisiones; g) los consejos se forman con responsables de diagnóstico y prescripción de la unidad, del nivel superior y del nivel inferior; h) los consejos reciben copias de los diversos reportes; e i) los consejos toman parte en decisiones y consideran impactos en todos los niveles.

\subsubsection{El subsistema de información y su impacto en la toma de decisiones.}

Las organizaciones «necesitan diferentes tipos de sistemas de información para sustentar la toma de decisiones y el trabajo operativo a diversos niveles y funciones organizacionales» (Laudon y Laudon, 2004, p. 38). De acuerdo con Gil y Giner,

Tener claro el sistema de información de base y apoyo a la empresa será básico para hacernos con las oportunidades que ofrece el poseer una información precisa, en tiempo real, cierta, en el momento oportuno y poco costosa, entre otras cualidades. (2012, p. 193)

Kendall y Kendall, por su parte, plantean que « todos los sistemas y subsistemas se interrelacionan y son interdependientes. Esta situación tiene importantes implicaciones tanto para las organizaciones como para los analistas de sistemas encargados de contribuir a que aquéllas consigan de la mejor manera sus metas» $(2005$, p. 28$)$. El subsistema de información se cristaliza en una organización en su MIS — management information system, por sus siglas en inglés-, el cual adquiere una diversidad de formas en las organizaciones y depende del sistema operativo en el cual se planea. Señala Bertalanffy que, «dado un determinado objetivo, encontrar caminos o medios para alcanzarlo requiere que el especialista en sistemas -o el equipo de especialistas- considere soluciones posibles y elija las que prometen optimización, con máxima eficiencia y mínimo costo en una red de interacciones tremendamente compleja» (2006, p. 2).

Sin embargo, el diseño del MIS parte de supuestos que los administradores emiten sobre las necesidades de información y de los posibles sucesos que requieran procesos o información diferente flexibilidad-: «Muchos de los errores cometidos aquí y allá pudieron haberse evitado si no se 
hubieran adoptado algunos supuestos falsos, que es común hacer y que generalmente están implícitos en las bases del diseño» (Ackoff, 2012, p. 284). En este sentido, Melchor, Lavín y Pedraza reconocen que,

la administración eficiente de los datos y la calidad en la información se considera un tema pendiente para todos los involucrados en los procesos de gestión, ya sean directivos, gerentes, personal de informática y cualquier usuario que hace uso día con día de la información generada por los sistemas. (2012, p. 139)
Álamo (2008) señala a tales sistemas con el nuevo concepto de «i-administración», o administración inteligente, $y$ la describe como aquella que utiliza las ventajas de las nuevas tecnologías y tiene la capacidad de prever y anticiparse a las demandas del usuario, de manera que responde a tiempo y con calidad. Esto es una administración sistémica individualizada que atiende de forma directa las necesidades del usuario, pero toma en cuenta el mercado y el entorno.

\section{Conclusiones y discusión}

$\mathrm{D}$ esde un punto de vista epistemológico, la administración ha sufrido distintas transformaciones con el paso del tiempo, entre las cuales se destacan dos grandes eras: «la mecánica», que se basa en los principios del análisis, la descomposición y el estudio de cada una de las partes a fin de comprender un todo. La anterior, en contraste con la «era de los sistemas», la cual se centra en el estudio de un «todo» al examinar la interacción y la sinergia de cada uno de los elementos que lo conforma. Este acervo de conocimiento histórico desarrollado establece la base fundamental de la actual ideología y metodología propuesta por los autores modernos de la administración como ciencia de estudio.

El modelo sistémico de Ackoff, utilizado como referente para comprender la aplicación del enfoque de sistemas en la compresión de la administración moderna, centra su paradigma en la interacción de las partes como un todo integrado a un sistema y a su vez en un suprasistema. Los distintos enfoques sobre la administración a lo largo del tiempo han construido de manera progresiva la vértebra de este enfoque moderno mediante la comparación de ideologías y postulaciones que enriquecieron el conocimiento sobre el tema. Incluso, desde tiempos de la gran civilización China, se establecían las bases del proceso administrativo moderno hace 2500 años con sus cuatro pasos: planeación, organización, liderazgo y control. Es por esto que autores como Fayol parten de estos principios del proceso administrativo y añaden el principio de «instrucción», pero transforman el principio de liderazgo en el principio actual de coordinación, el cual se define como el proceso sistémico que integra todas las actividades y departamentos de la empresa, de modo que facilita su trabajo para la obtención de mejores resultados. Con esto se aportan, sin duda, elementos iniciales al actual enfoque sistémico.

La Revolución Industrial marcó un parteaguas en la sociedad a nivel mundial, $\mathrm{y}$ con esta vinieron distintos cambios en la administración debido al proceso de producción en masa. El enfoque administrativo de producción y centrado en las máquinas, más que en las personas, fue severamente criticado y propició que investigadores de la materia surgieran con un nuevo método que les permitiera dirigir de forma eficaz los esfuerzos de cada persona dentro de la organización. Taylor hizo la primera aportación al proponer los nuevos principios administrativos, con lo cual nace la administración científica, pero su teoría requirió de otros autores que enfocaron sus investigaciones para incluir en sus modelos la atención de factores sociales y psicológicos. De esta manera surgen las investigaciones contemporáneas y humanistas que incorporan factores como, por ejemplo, el compromiso del empleado y la pertenencia a la organización, la 
atención del entorno laboral como factor motivante del trabajo y, por supuesto, la satisfacción personal del empleado y, por ende, de sus necesidades personales y sociales. En este proceso el enfoque de contingencias aportó un elemento fundamental para el enfoque actual sistémico al incluir como elemento determinante la situación vigente en el momento administrativo y sus condiciones inesperadas.

Es por esto que el enfoque sistémico integra todos los elementos de las escuelas humanistas y de contingencias, así como mejora su modelo administrativo al considerar la analogía del medio ambiente en el que declara que no existen elementos químicos independientes, y que todos los elementos se relacionan de manera interdependiente en cada una de sus partes, lo cual causa impactos en subsistemas, sistemas y suprasistemas. Ackoff se sumó a esta visión al establecer que cualquier cambio puede mejorar de forma significativa la eficiencia; sin embargo, cuando todos los cambios se llevan a cabo juntos existe un efecto multiplicador que supera a la suma de las partes. Esta, sin duda, es la tesis fundamental de la aplicación del modelo de sistemas al ámbito administrativo, según la cual debe existir una correlación en la que actúen de manera simultánea y con sinergia cada una de las partes a fin de alcanzar algún objetivo establecido. Por tanto, se puede comprender que el nuevo enfoque sistémico es el resultado de un proceso de evolución de la teoría administrativa, así como del análisis de la adaptación y la tropicalización de cada escuela administrativa con respecto a las necesidades de cada época, pero con una visión de futuro que conduce a una utopía con cierto grado de realismo.

\section{Referencias}

Ackoff, R. (2012). El paradigma de Ackoff: Una administración sistémica. México: Limusa Wiley.

Ahoy, C. (2010). Administración de operaciones con enfoque al cliente: cómo alinear los procesos de negocios y las herramientas de calidad para alcanzar la efectividad operativa. México: $\mathrm{McGraw-Hill} \mathrm{Interamericana.}$

Álamo, Ó. D. (2008). La profecía de la administración inteligente. IDP. Revista de Internet, Derecho y Política, 6, 1-14. Recuperado de http://www.redalyc.org/articulo. oa?id=78817209002

Ansoff, H. I.; Declerk, R. P.; Hayes, R.L. (2003).El planteamiento estratégico: nueva tendencia de la administración. México: Editorial Trillas.

Argyris, C. (2001). Sobre el aprendizaje organizacional. México: Oxford University Press.

Arias, M. C.; Del Toro, M.P.; Guerrero, J.C. (2013). Administración: evolución y cambios en una sociedad cambiante. Global Conference on Business \& Finance Proceedings, 8(2), 1613-1617.

Bartol, K. M.; Martin, D. C. (1998). Management. Estados Unidos: McGraw-Hill.

Bateman, T. S.; Snell, S.A. (2009). Administración, liderazgo y colaboración en un mundo competitivo. México: McGraw-Hill.
Bertalanffy, L. V. (2006). Teoría general de los sistemas. México: Fondo de Cultura Económica.

Bolman, L. G.; Deal, T. E. (1984). Modern approaches to understanding and managing organizations. San Francisco, Estados Unidos: Jossey-Bass Publishers.

Certo, S. C. (2001). Administración moderna. Bogotá: Prentince Hall.

Chiavenato, I. (2005). Administración de los nuevos tiempos. Bogotá: McGraw Hill.

Contreras, M. R.; Benítez, J. L. (2011). La toma de decisiones y el cambio en la organización local. En C. Gutiérrez, D. C. Caldera y J. A. Martínez (Eds.), Avatares del Estudio de las Organizaciones. Tomo 2: estudios de caso (173-188). México: Editorial Fontamara.

Csikszentmihalyi, M. (1998). Creatividad: el fluir y la psicología del descubrimiento y la invención. España: Paidós.

Daft, R. (2004).Administración. México: International Thomson Editores.

Debernardo, H.; Hurtado, M. (2006). El puente: mejores los resultados de su empresa aplicando el pensamiento sistémico. Buenos Aires: Granicas.

Drucker, P. F. (2006). Drucker para todos los días. Colombia: Grupo Editorial Norma. 
Etkin, J. R. (2005). Gestión de la complejidad en las organizaciones. Argentina: Ediciones Granica.

Eyssautier, M. (2002). Elementos básicos de administración. México: Editorial Trillas.

Fretes, G. (2009). Fundamentos de administración empresarial. Argentina: El Cid Editor.

Garza, J. G. (2003). Administración contemporánea. México: McGraw-Hill.

Gil, M. A.; Giner, F. (2012). Cómo crear y hacer funcionar una empresa. México: Alfaomega Grupo Editor.

Gómez-Mejía, L. R.; Balkin, D.B.; Cardy, R.L. (2008). Management: people, performance, change. Estados Unidos: McGraw-Hill, Irwin.

Hampton, D. (2003). Administración. México: McGraw-Hill Interamericana.

Hellriegel, D.; Jackson, S. E.; Slocum, J. W. (2010). Administración. Un enfoque basado en competencias. México: Cengage Learning.

Hernández, R. (2008). Administración teoría, proceso, áreas funcionales y estrategias para la competitividad. México: McGraw-Hill Interamericana.

Herrscher, E. (2009). Planeamiento sistémico: un enfoque estratégico en la turbulencia. México: Argentina Garnica.

Johansen, B. O. (2004). Introducción a la teoría general de sistemas. México: Limusa-Noriega Editores.

Jones, G.; George, J. (2010). Administración contemporánea. México: McGraw-Hill Interamericana.

Kast, F. E.; Rosenzweig, J.E. (1987). Administración en las organizaciones. Enfoque de sistemas y de contingencias. México: McGraw-Hill.

Kendall, K. E.; Kendall, J. E. (2005). Análisis y diseño de sistemas. México: Pearson.

Koontz, H.; Weihrich, H. (1998). Administración una perspectiva global. México: McGraw-Hill.

Laudon, K.; Laudon, J. P. (2004). Management information systems: managing the digital firm. Estados Unidos: Pearson Prentince Hall.
López, M. E.; Arias, L.; Rave, S. N. (2006). Las organizaciones y la evolución administrativa. Scientia Et Technica, XII(31) 147-152. Recuperado de http://www.redalyc.org/articulo. oa? $\mathrm{id}=84911639026$

Majluf, N. (2011). Los desafíos de la gestión: de lo formal a lo sutil. Chile: El Mercurio.

Melchor, J.; Lavín, J.; Pedraza, N. A. (2012). Seguridad en la administración y calidad de los datos de un sistema de información contable en el desempeño organizacional. Contaduría y Administración, 57(4), 11-34. Recuperado de http://148.215.2.10/articulo.oa?id=39524375002

Meléndez, M.Á.; Solís, P. C.; Gómez,J. G. I. (2010). Gobernanza y gestión de la universidad pública. Revista de Ciencias Sociales (Ve), XVI(2), 210-225. Recuperado de http://www. redalyc.org/articulo.oa?id=28016298003

Merril, H. F. (1999). Clásicos en administración. México: Editorial Limusa.

Mintzberg, H. (2001). Diseño de organizaciones eficientes. Argentina: Editorial El Ateneo.

Ramírez, C. (2009). Fundamentos de administración. Bogotá: Ecoe Ediciones.

Ríos, L. G.; Mustafá, Y. (2004). Algunas analogías entre los sistemas de ingeniería y los sistemas administrativos. Scientia Et Technica, 10(25), 175-178. Recuperado de http://www. redalyc.org/articulo.oa?id=84911685031

Savall, H.; Zardet, V.; Bonnet, M. (2008). Mejorar los desempeños ocultos de las empresas a través de una gestión socioeconómica. Italia: OIT-Iseor.

Valdés, L. A. (2005). Nota técnica. Aplicaciones administrativas empíricas del análisis estructural de los sistemas en la toma de decisiones. Contaduría y Administración, 217, 149-179. Recuperado de http://www.redalyc.org/articulo. oa? id $=39521707$

Varela, R. A. (2013). Administración de la compensación: sueldos, salarios y prestaciones. México: Pearson.

Velásquez, V. F. (2000). El enfoque de sistemas y de contingencias aplicado al proceso administrativo. Estudios Gerenciales, (77), 27-40. 